\title{
Analyse de la mise en place d'une aide individuelle à l'écriture du premier script dans un portfolio pour des étudiants du diplôme d'études spécialisées en médecine générale
}

\author{
Implementation of individual assistance in drafting the first script \\ of a general practice postgraduate portfolio
}

Mélanie AFONSO ${ }^{1}$, Shérazade KINOUANI ${ }^{1}$, Jean-Louis DEMEAUX ${ }^{1}$ et Bernard GAY ${ }^{1}$

1 Département de Médecine Générale, Université de Bordeaux, 146 rue Léo Saignat, Case 148, 33076 Bordeaux cedex, France

Manuscrit soumis à la rédaction le 18 août 2013 ; commentaires éditoriaux formulés aux auteurs le 5 novembre 2013 et le 3 février 2017 ; accepté pour publication le 6 février 2017

\author{
Mots-clés \\ portfolio ; tutorat ; \\ médecine générale ; \\ apprentissage ; \\ compétences
}

Résumé - Contexte : Le portfolio est utilisé dans la formation et la validation du diplôme d'études spécialisées de médecine générale à l'Université de Bordeaux depuis 2004. L'appropriation de cet outil restait difficile pour les étudiants, ce qui a conduit le Département de médecine générale (DMG) à proposer une aide individuelle à l'écriture du premier script du portfolio en 2010. Objectif : Analyser l'usage de l'aide individuelle à l'écriture du premier script auprès des étudiants de première année de troisième cycle et des enseignants impliqués dans le dispositif. Méthode : enquête déclarative par questionnaires à l'attention des étudiants et des enseignants en 2012. Résultats : Le taux de réponse a été de $70 \%$ chez les étudiants et de $44 \%$ chez les enseignants. Soixante-douze pour cent des étudiants étaient intéressés par cette aide et $86 \%$ d'entre eux avaient envoyé leur premier script à un enseignant désigné. Le temps de rétroaction avec les enseignants a été jugé insuffisant par $74 \%$ des étudiants et $81 \%$ souhaiteraient une aide complémentaire. Plus de $65 \%$ trouvaient un intérêt à l'aide apportée mais $31 \%$ se sentaient encore en difficulté au décours. Chez les enseignants, $88 \%$ estimaient que l'aide était partiellement suffisante et que $12 \%$ des étudiants étaient motivés. Conclusion : Le dispositif était perçu comme une bonne initiative mais l'adhésion restait dépendante du lien tissé entre l'étudiant et l'enseignant. Des modifications ont été apportées au dispositif. Le DMG a notamment mis en place un e-portfolio qui fera l'objet de prochaines études. 


\section{Keywords} portfolio; mentoring; general practice; learning skills

\begin{abstract}
Background: The portfolio used at the University of Bordeaux since 2004 has a double purpose: the learning and the assessment of the postgraduate general medicine degree. Postgraduate students found it hard to suit the portfolio to their needs, which is why the teachers of the general practice department set up individual mentoring to help students draft the first script. Goal: To analyze the individual mentoring process among first-year postgraduate students and teachers involved in the project. Method: Declarative survey questionnaires filled in by the involved students and teachers. Results: The response rate for students was $70 \%$ and for teachers $44 \%$. Some $72 \%$ of students were interested in the mentoring and $86 \%$ returned their first script to their appointed teacher. Some $74 \%$ of students felt that the time spent with teachers was inadequate and $81 \%$ would have liked further assistance. Over $65 \%$ found the assistance helpful, but $31 \%$ still felt uncomfortable with script writing after the mentoring. Some $88 \%$ of teachers felt that the one-time mentoring had been partly satisfactory and that $12 \%$ of students were motivated. Conclusion: The process was considered helpful but adherence depended on teacher-student relationship. The department has made some changes in the process and set up the ePortfolio, which will be the subject of future studies.
\end{abstract}

\section{Introduction}

Le portfolio est, depuis plusieurs années, un outil très largement utilisé par les facultés de médecine françaises pour la formation, l'évaluation et la validation au cours du diplôme d'études spécialisées (DES) de médecine générale ${ }^{[1,2,3]}$. Il est reconnu pour ses valeurs formatives d'aide à l'acquisition de compétences, au moyen de tâches d'apprentissage évolutives au cours du DES. Il est utilisé depuis 2004 à l'Université de Bordeaux à des fins formative et sommative ${ }^{[4]}$. Au cours des trois ans de spécialisation, les internes doivent inclure dans leur portfolio deux types de documents narratifs : d'une part, douze récits de situations complexes authentiques (appelés localement scripts) ; d'autre part, dix récits de situations simples authentiques (appelés situations ponctuelles). La rédaction de telles contributions confronte les internes à un certain nombre de difficultés, qui ont justifié la mise en place d'un dispositif d'accompagnement spécifique.

\section{Contexte et problématique}

Le script correspond à une situation clinique complexe ayant posé problème à l'étudiant. Il l'expose dans son portfolio selon un format imposé. La présentation du script est composée de cinq parties, que sont respectivement le récit, les tâches d'apprentissage et les compétences acquises, la synthèse et les références. Le récit décrit une situation où l'interne a été en difficulté du fait de sa complexité. Il rend compte des faits tels que les a perçus l'interne, sans omission volontaire. Il doit inclure les éléments sémiologiques et biographiques du patient, le contexte de la rencontre, les problèmes posés. Les aspects émotionnels et le ressenti de l'interne doivent être explicités ainsi que les interactions relationnelles avec le patient et l'environnement (qu'elles soient facilitatrices ou bloquantes). Le récit relate ensuite les stratégies de résolution de problèmes et les décisions finalement prises. L'analyse est un travail réalisé à distance de la situation. Elle a pour but d'expliciter et de critiquer de façon réflexive le raisonnement clinique et la démarche décisionnelle. Cette analyse s'appuie sur les trois composantes de l'EvidenceBased-Medicine, à savoir les données probantes disponibles, issues notamment de la recherche clinique, l'expérience du clinicien et les préférences du patient. Il s'agit de la partie du script dans laquelle l'interne procède à son auto-évaluation et oriente ses apprentissages. Les tâches d'apprentissage et les compétences acquises font l'objet d'une troisième partie. L'interne y décrit et documente les compétences 
acquises à partir de la situation. Il programme aussi les tâches d'apprentissage nécessaires à l'acquisition des compétences ayant pu manquer. La synthèse reprend les points essentiels de l'auto-évaluation et de l'auto-formation. L'interne y décrit l'impact du travail d'écriture en termes d'apprentissage et les éventuelles modifications à envisager pour sa pratique future. Enfin, les références exploitées en tant que ressources lors de la démarche sont colligées.

La situation ponctuelle correspond à une situation vécue plus simple. L'interne rapporte succinctement la situation vécue et le problème rencontré. Il expose ensuite de façon synthétique la réponse trouvée à ce problème après une recherche documentaire. La situation ponctuelle est une tâche d'écriture dont le format ne doit pas dépasser une page et une ou au maximum deux références.

Le type de portfolio qui résulte de l'incorporation de telles contributions peut être considéré comme semi-sélectif. L'interne a le libre choix des situations relatées (scripts et situations ponctuelles). Il peut aussi ajouter dans son portfolio d'autres documents s'il estime qu'ils reflètent ses expériences de formation et sa progression : ce sont les contributions diverses. Cependant, l'interne constitue son portfolio en respectant le format imposé décrit ci-dessus. Tout au long du cursus, il devra aussi présenter ces scripts lors de séances de cours qui sont en partie thématisées. Ces séances ont lieu environ une fois par mois. Il s'agit d'ateliers en groupe de 15 à 25 internes, en présence d'un enseignant. Ce formateur a la tâche de fournir une rétroaction individualisée à l'interne sur le script présenté, en amont ou en aval de la séance. Par ailleurs, dans une optique sommative et certificative, le Département de médecine générale (DMG) exploite ces différentes sources d'informations en tant que traces d'apprentissage : il se prononce en effet sur l'acquisition des compétences du médecin généraliste décrites dans le référentiel métier, à partir d'une appréciation du contenu du portfolio, et notamment des contributions prescrites que sont les scripts et les situations ponctuelles, de leur présentation en cours et des informations échangées lors des moments de rétroaction autour du script.

Différents types d'accompagnement des internes ont été testés au sein du programme de DES de médecine générale à l'Université de Bordeaux depuis la mise en place du portfolio, dans une logique d'approche pédagogique par compétences. En 2005, devant la difficulté des étudiants à s'approprier le nouvel outil, le DMG a proposé un tutorat individuel. Cependant, les responsables du DMG ont constaté que les étudiants sollicitaient insuffisamment cette forme d'assistance. De plus, ceux qui demandaient un tuteur n'étaient pas forcément ceux qui étaient le plus en difficulté En 2008, le tutorat individuel obligatoire a été suspendu devant l'augmentation croissante du nombre d'internes dans la région, engendrant un coût financier du dispositif ainsi qu'une charge de travail pour les enseignants trop importants. Un autre type d'accompagnement a alors été mis en place : le tutorat collectif. Il consiste à apporter des réponses aux questions des internes relatives au portfolio et à sa construction lors de séances en groupes. Ces séances, appelés séances d'analyse du portfolio, ont lieu une à deux fois par semestre. Elles durent chacune deux heures, en présence d'un ou deux enseignants, selon la taille du groupe.

Devant les difficultés des étudiants, le DMG a ensuite complété ce tutorat collectif en 2010 d'une aide individuelle à l'écriture du premier script, proposée aux internes au cours des quatre premiers mois de leur DES. Chaque étudiant doit adresser, au milieu du premier semestre, un script à un enseignant désigné, appelé enseignant référent. Les étudiants et enseignants ont pour consigne de privilégier des interactions de nature présentielle mais le cadre reste souple pour laisser le choix aux protagonistes de recourir à d'éventuelles interactions asynchrones, par exemple par courrier électronique. L'enseignant référent analyse avec l'étudiant la production fournie et l'aide à apporter des corrections. Les objectifs de cette aide sont les suivants : expliquer à l'étudiant la finalité du travail d'écriture de script et du portfolio ; rendre explicite ce que sont les soins de santé primaires, en tant que cadre conceptuel pour son analyse réflexive de pratique ; l'aider à identifier les situations vécues propices à l'écriture d'un script ; l'aider dans l'appropriation de la démarche réflexive ; l'aider à s'approprier le format imposé du portfolio.

L'objectif de cette étude était d'analyser l'usage de ce dispositif de la part des étudiants de première 
année de DES et des enseignants de médecine générale impliqués.

\section{Méthodes}

Le travail a été effectué dans le cadre de la thèse pour le diplôme d'état de docteur en médecine de l'auteure principale de l'article. Il s'agit d'une enquête transversale déclarative auprès des étudiants et des enseignants.

Concernant le volet étudiant de l'enquête, une étude de faisabilité a été conduite auprès des étudiants de première année du DES au cours de l'année universitaire 2010-2011, au moyen d'un questionnaire auto-administré envoyé par courriel, avec plusieurs relances. Devant le faible taux de réponses à l'étude de faisabilité (29\%), une autre stratégie de sollicitation a été adoptée pour l'étude au cours de l'année universitaire 2011-2012. Le questionnaire a été modifié à la lecture des réponses obtenues pour en améliorer la pertinence et la compréhension. Le questionnaire remanié était anonyme. Il a été distribué aux étudiants de première année du DES sous format papier en amphithéâtre au cours de la séance du choix des stages pour le second semestre. Ce choix a eu lieu le 30 Mars 2012. La distribution s'est faite en début du choix de stage, après qu'une information orale ait été donnée au micro par le coordonnateur du DES et l'auteure de l'enquête. La nécessité de remplir le questionnaire et de le remettre lors de la sortie de l'amphithéâtre dans les boîtes dédiées a été expliquée. Les étudiants avaient toute la durée du choix de stage pour remplir le questionnaire. Une notice d'information garantissant l'anonymat des données était jointe au questionnaire.

Deux autres questionnaires étaient destinés aux enseignants référents (ER) et aux responsables des commissions pédagogiques locales (CPL). La subdivision administrative d'internat de Bordeaux a été découpée par le DMG en 10 territoires appelés CPL. Ces territoires correspondent à des zones géographiques où des internes réalisent leurs stages ambulatoires et hospitaliers. Les responsables des CPL assurent la coordination locale des séances d'enseignement. Ils sont aussi les intermédiaires entre les enseignants locaux et les étudiants. Ils ont tous reçu les questionnaires anonymes en pièce jointe à un courriel. Le renvoi des questionnaires pouvait être fait par retour de courriel, par courrier postal ou par fax. Trois relances ont été effectuées. Le choix de la voie électronique pour l'envoi des deux questionnaires s'est imposé du fait de l'impossibilité de mobiliser les enseignants et les responsables de CPL en réunion en même temps et au même endroit. En effet, ils sont répartis sur l'ensemble de la région qui s'étend sur plus de $41000 \mathrm{~km}^{2}$.

Les trois questionnaires ont été élaborés par l'auteure de la thèse et son directeur de thèse. Leur pertinence a été validée de façon consensuelle par les enseignants du DMG, après réalisation de l'étude de faisabilité. Le questionnaire étudiant comportait 25 items et une zone d'expression libre (tableau I). Celui destiné aux enseignants référents comptait 23 items (tableau II).Tous deux portaient sur : la perception du portfolio et du script à l'entrée en DES ; l'intérêt et l'organisation du dispositif ; les aspects abordés au cours de l'aide individuelle et la satisfaction générale au décours. Le questionnaire pour les responsables de CPL contenait 10 items relatifs à la supervision du dispositif (tableau III).

La saisie et l'analyse statistique des données ont été réalisées au moyen du logiciel Epi-info (version 3.5.1 Août 2008). Les données quantitatives ont été décrites à l'aide de moyennes, les données catégorielles à l'aide de fréquences. Les réponses aux questions ouvertes ont été relevées dans le cadre d'un processus de catégorisation thématique, à l'aide de « phrases-clés » regroupées sous forme de tableaux.

Ce travail s'est inscrit dans une démarche qualité d'évaluation des outils pédagogiques. ${ }^{[5]}$

\section{Résultats}

\section{Enquête auprès des étudiants}

Sur les 215 étudiants inscrits en première année de DES, 194 étaient présents le jour du choix. Parmi eux, 139 ont accepté de participer à l'étude, soit un taux de participation de 71,6\%. 
Tableau I. Questionnaire à l'attention des étudiants.

\begin{tabular}{|c|c|}
\hline QUESTIONS & REPONSES \\
\hline $\begin{array}{l}\text { Les informations que vous avez reçues pendant le } 1^{\text {er }} \text { cours du DES vous ont-elles } \\
\text { semblé suffisantes pour réaliser un script }\end{array}$ & Echelle de Likert \\
\hline $\begin{array}{l}\text { Vous sentiez vous en difficulté face au Portfolio et à la façon dont il fallait aborder } \\
\text { l'écriture d'un script? }\end{array}$ & Echelle de Likert \\
\hline Vous êtes-vous senti intéressé par cette aide à l'écriture du premier script ? & Echelle de Likert \\
\hline Vous a-t-il été difficile de joindre votre enseignant réfèrent? & Echelle de Likert \\
\hline Y a-t-il eu une rencontre en dehors des réponses par mail? & Oui/Non \\
\hline Quelle a été la nature de votre rencontre (plusieurs réponses possibles) ? & Téléphonique/Présentiel \\
\hline Nombre d'entretien(s) réalisé(s) ? & 0 à $>2$ \\
\hline Temps moyen passé avec l'enseignant référent ? & $<15 \min$ à $>1$ heure \\
\hline L'aide apportée a-t-elle été suffisante en termes de temps? & Echelle de Likert \\
\hline A ce propos, l'enseignant référent vous a-t-il adressé la grille d'évaluation? & Oui/Non \\
\hline Cette grille vous a-t-elle paru utile? & Echelle de Likert \\
\hline Item(s) sur lequel (s) le plus de temps a été passé ? & Choix Multiples \\
\hline $\begin{array}{l}\text { Correspondaient-ils à ceux qui vous ont posé le plus de souci lors de l'écriture du } \\
\text { script? }\end{array}$ & Echelle de Likert \\
\hline Thème(s) que vous auriez souhaité voir abordé(s) ? & Choix Multiples \\
\hline Autres ? & Ouverte \\
\hline $\begin{array}{l}\text { En dehors du script et du portfolio avez-vous pu discuter avec votre enseignant } \\
\text { référent d'autres thèmes concernant la médecine générale et son enseignement? }\end{array}$ & Oui/Non \\
\hline Si oui, lesquels? & Ouverte \\
\hline Trouvez-vous à posteriori de l'intérêt pour cette initiative ? & Echelle de Likert \\
\hline Comprenez-vous mieux dorénavant ce qu'il est attendu dans un script ? & Echelle de Likert \\
\hline Vous sentez-vous désormais plus à l'aise pour la réalisation d'un second script? & Echelle de Likert \\
\hline Seriez-vous intéressé par une aide complémentaire? & Echelle de Likert \\
\hline Etes-vous plus motivé pour réaliser des scripts à l'issue de ce projet? & Oui/Non \\
\hline Si oui pourquoi ? & Ouverte \\
\hline Si non pourquoi ? & Ouverte \\
\hline Commentaires libres & Ouverte \\
\hline
\end{tabular}

Une proportion de $85,6 \%$ des internes avaient renvoyé leur premier script à leur ER et $71,9 \%$. déclaraient un intérêt pour cette initiative. Ils se disaient en difficulté par rapport à l'écriture des scripts dans $63,9 \%$ des cas. Les informations sur le portfolio reçues pendant le premier cours de DES semblaient insuffisantes pour 33,1\% des étudiants et moyennement suffisantes pour $53,2 \%$ d'entre eux.

Concernant les interactions avec l'ER, 68,4\% avaient eu des échanges exclusivement par courriel.
Pour les autres, l'entretien avait été téléphonique $(69 \%)$ ou présentiel (31\%). Ces résultats sont présentés dans le tableau IV. La moitié des étudiants déclarait avoir rencontré de grandes difficultés pour joindre leur ER.

Le temps passé par l'étudiant avec l'ER est décrit dans le tableau V. Ce temps ne leur semblait pas totalement suffisant dans $73,8 \%$ des cas.

Les étudiants déclaraient avoir vu et/ou rempli la grille d'évaluation formative relative au script à 
Tableau II. Questionnaire à l'attention des enseignants référents.

\begin{tabular}{|c|c|}
\hline QUESTIONS & REPONSES \\
\hline Cette aide à l'écriture du $1^{\mathrm{er}}$ script vous a-t-elle semblé être une bonne initiative ? & Oui/Non \\
\hline $\begin{array}{l}\text { Aviez-vous perçu d'éventuelles difficultés émanant des étudiants avant } \\
\text { l'instauration de cette aide (notamment durant les leres séances de cours)? }\end{array}$ & Oui/Non \\
\hline Combien d'étudiants aviez-vous en charge? & Nombre \\
\hline $\begin{array}{l}\text { Combien vous ont-ils contacté dans le délai imparti de } 15 \text { jours après le premier } \\
\text { cours? }\end{array}$ & Nombre \\
\hline Combien d'étudiants avez-vous été contraint de solliciter vous-même ? & Nombre \\
\hline Combien d'étudiants vous ont renvoyé un script finalement? & Nombre \\
\hline Les étudiants ont-ils alors été facilement joignables ? & Oui/Non \\
\hline Combien de temps vous, en moyenne, passé avec les étudiants? & $<15$ min à $>1$ heure \\
\hline Quel type de rencontre avez-vous privilégié ? & Mail/Téléphone/Présentiel \\
\hline Pour quelle raison ? Etait-ce en fonction de la qualité du script produit ? & Ouverte \\
\hline Temps moyen passé par étudiant sur l'ensemble du processus ? & $<30$ min à $>2$ heures \\
\hline Quel a été le thème le plus abordé avec les étudiants ? & Choix Multiples \\
\hline Quel(s) a (ont) été le(s) thème(s) le(s) moins bien compris ? & Choix Multiples \\
\hline Avez-vous utilisée la grille d'évaluation comme support auprès des étudiants ? & Oui/Non \\
\hline Pour combien des étudiants que vous aviez en charge ? & Nombre \\
\hline $\begin{array}{l}\text { Avez-vous abordé d'autres thèmes que le script avec les étudiants (la médecine } \\
\text { générale, les études, la recherche, les perspectives d'avenir...) }\end{array}$ & Ouverte \\
\hline Avez-vous perçu un engouement des étudiants envers cette initiative ? & Echelle de Likert \\
\hline L’aide apportée vous a-t-elle semblée suffisante pour les étudiants ? & Echelle de Likert \\
\hline Quels sont les aspects positifs d'une telle aide à vos yeux ? & Ouverte \\
\hline Quels en sont les aspects négatifs ? & Ouverte \\
\hline Quelles améliorations y apporteriez-vous? & Ouverte \\
\hline Souhaiteriez-vous participer de nouveau l'année prochaine? & Oui/Non \\
\hline Quelle est votre commission pédagogique locale d'enseignement ? & Ouverte \\
\hline
\end{tabular}

l'issue de la rétroaction avec l'ER dans 48,5\% des cas. Cette grille semblait moyennement utile pour $42,5 \%$ d'entre eux et très utile pour $39,7 \%$ (tableau VI).

Aucun autre thème que le portfolio n'était abordé dans $87,8 \%$ des échanges. Si d'autres thèmes étaient abordés, il s'agissait du cursus de médecine générale, des diplômes d'études spécialisées complémentaires (DESC), de l'installation, des stages chez le praticien, de la pratique en cabinet, des cours, des maisons médicales et de la sécurité sociale.

A l'issue des quatre mois du dispositif, 30,8\% des étudiants se disaient encore en difficulté par rapport à ce qui était attendu, tandis que 69,2\% avaient partiellement trouvé leurs réponses. Quant à l'écriture du second script, 68,5\% se sentaient mieux armés pour s'y atteler tandis que $31,5 \%$ se sentaient encore en difficulté.

L'éventualité d'une aide complémentaire était favorablement perçue par 80,6\% des étudiants. Dans $75,6 \%$ des cas, ils n'étaient pas plus motivés pour l'écriture des scripts suivants.

Parmi les aspects positifs déclarés par les étudiants au sujet de l'aide à l'écriture et du portfolio, sont rapportés une meilleure compréhension de ce 
Tableau III. Questionnaire à l'attention des responsables de commissions pédagogiques locales.

\begin{tabular}{|l|l|}
\hline QUESTIONS & REPONSES \\
\hline Combien d'enseignants référents aviez-vous en charge ? & Nombre \\
\hline Leur implication vous a-t-elle semblée satisfaisante ? & Echelle de Likert \\
\hline Verriez-vous un moyen de l'améliorer davantage ? & Ouverte \\
\hline Avez-vous été sollicité par les ER pour obtenir de l'aide ou des renseignements ? & Oui/Non \\
\hline Si oui, sur quels thèmes ? & Ouverte \\
\hline $\begin{array}{l}\text { Avez-vous pris contact avec vos enseignant référent au cours de l'initiative pour } \\
\text { faire le point sur l'avancée de leurs analyses de script? }\end{array}$ & Oui/Non \\
\hline Pour quelles autres raisons? & Ouverte \\
\hline $\begin{array}{l}\text { Avez-vous eu des appels d'étudiants pour des soucis de joignabilité ou d'entente } \\
\text { avec leur enseignant référent? }\end{array}$ & Oui/Non \\
\hline Avez-vous été en difficulté par rapport à votre rôle ? Pour quelles raisons ? & Ouverte \\
\hline Verriez-vous des améliorations à apporter l'an prochain ? & Ouverte \\
\hline
\end{tabular}

Tableau IV. Types d'interaction entre les étudiants et les enseignants, déclarés par les internes de première année de l'Université de Bordeaux Mars 2012, $n=139$.

\begin{tabular}{|l|l|}
\hline $\begin{array}{l}\text { Type d'interaction pour l'aide à l'écriture } \\
\text { du } 1^{\text {er }} \text { script }\end{array}$ & Pourcentage \\
\hline Mail & 68,4 \\
\hline Téléphonique & 21,8 \\
\hline Présentiel & 9,8 \\
\hline
\end{tabular}

Tableau V. Temps déclaré passé lors de l'interaction par les étudiants $(n=139)$ et par les enseignants référents $(n=17)$. Mars 2012.

\begin{tabular}{|l|l|l|}
\hline $\begin{array}{l}\text { Temps passé entre étudiants et } \\
\text { enseignants référents (ER) }\end{array}$ & $\begin{array}{l}\text { Etudiants } \\
(\%)\end{array}$ & ER(\%) \\
\hline Moins de 15 minutes & 81,4 & 35,3 \\
\hline Entre 15 et 30 minutes & 10,8 & 41,2 \\
\hline Entre 31 minutes et 1 heure & 3,9 & 5,9 \\
\hline Plus de 1 heure & 3,9 & 17,9 \\
\hline
\end{tabular}

qu'il était attendu d'un script $(13,8 \%)$ et la prise de recul par rapport à la prise en charge $(5,5 \%)$. Parmi les freins à l'écriture étaient évoqués, entre autres, l'aspect chronophage de la rédaction $(33,3 \%)$, le manque d'intérêt pour le portfolio $(20,8 \%)$, la difficulté d'adaptation aux soins primaires de situations vécues à l'hôpital $(30,5 \%)$ et le manque de retour des enseignants $(12,5 \%)$.

\section{Enquête auprès des enseignants référents}

Le taux de participation a été de 43,6\% (17/39).

Chaque ER avait à sa charge entre quatre et huit étudiants. Les ER avaient reçu un premier script de 77,3\% des étudiants : 23,6\% des ER avaient reçu les scripts dans les temps impartis, $41,2 \%$ disaient ne pas avoir été contactés par les étudiants avant le délai fixé par le dispositif. La totalité des ER répondeurs estimait que le dispositif était une bonne initiative. Ils percevaient des difficultés quant à la rédaction des scripts chez les étudiants dans $82,4 \%$ des cas.

Dans $75 \%$ des cas, les ER avaient été contraints de joindre eux-mêmes au moins un des étudiants dont ils avaient la charge. Les étudiants étaient facilement joignables dans $76,5 \%$ des cas.

Le temps passé avec les étudiants déclaré par les ER est présenté dans le tableau V.

Les ER percevaient les étudiants comme motivés dans $11,8 \%$ des cas, peu ou pas intéressés pour $58,8 \%$ d'entre eux. Ils estimaient que l'aide mise en place n'était que partiellement suffisante dans $88,2 \%$ des cas. 
Tableau VI. Grille d'évaluation formative.

1 : insuffisant $2:$ moyen $3:$ bon $4:$ excellent

\begin{tabular}{|l|l|l|l|}
1 & 2 & 3 & 4
\end{tabular}

Forme :

Les quatre parties du script (récit, analyse, tâches induites, synthèse) sont présentes et distinctes les unes des autres

Auto apprentissage : les traces de la recherche documentaire sont présentes

Fond :

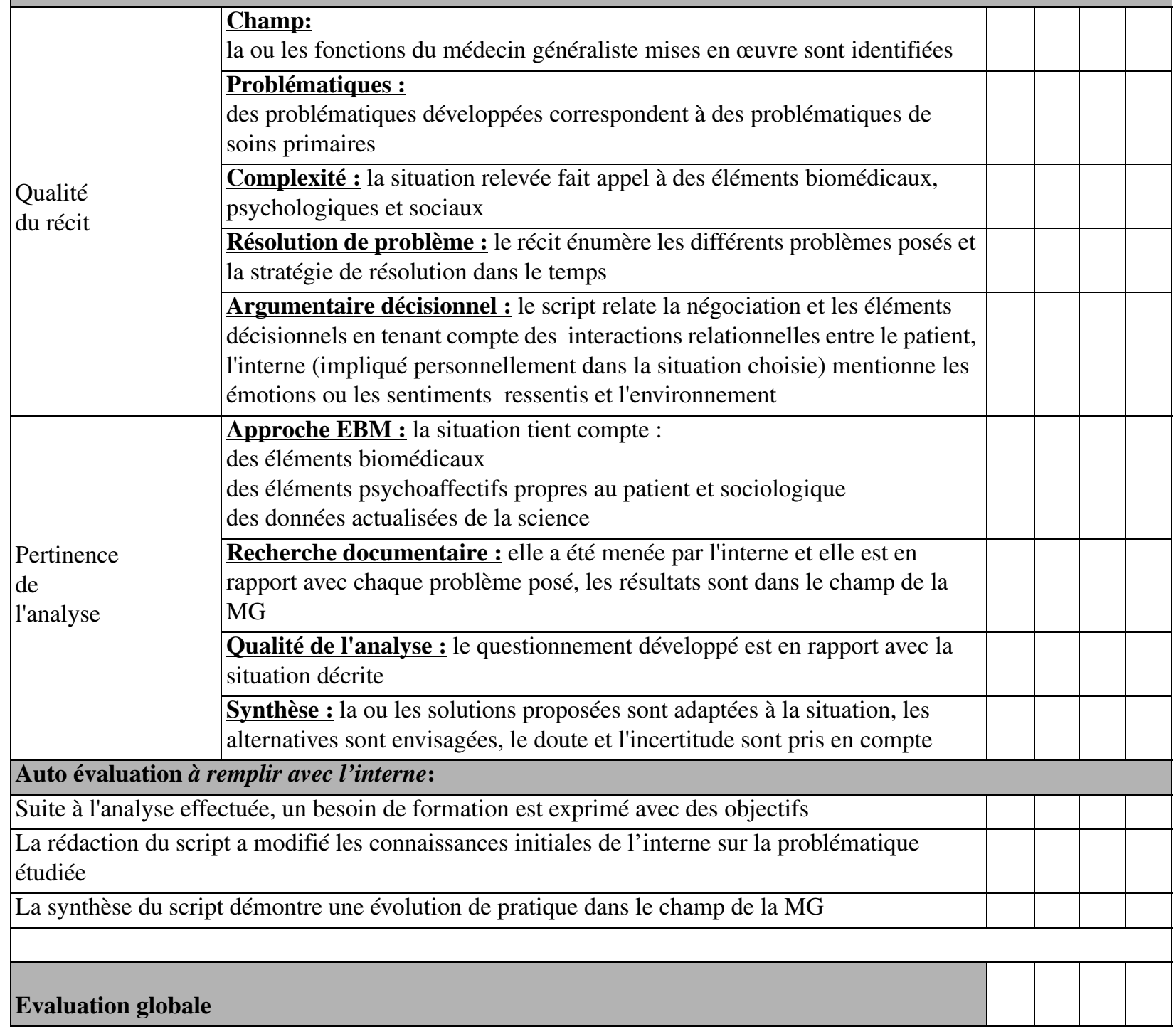

Les aspects positifs du dispositif décrits par les ER étaient les suivants :

- compréhension plus rapide de ce qui est attendu d'un script ;
- meilleure appropriation de l'outil et limitation de l' « opposition frontale au portfolio »;

- mise en place d'objectifs clairs dès le début du cursus ; 
- entrée favorisée dans un processus pédagogique continu.

Les freins relevés par les ER étaient les suivants :

- importante charge de travail supplémentaire ;

- manque de motivation des étudiants ne comprenant pas l'intérêt de l'aide ;

- difficultés d'organisation des rencontres présentielles du fait de l'éloignement géographique.

Enquête auprès des responsables de commissions pédagogiques locales

Quatre responsables de CPL sur les neuf ont répondu au questionnaire $(44,4 \%)$.

Les responsables de CPL ressentaient une implication des ER dans $50 \%$ des cas. Ils avaient été sollicités par des ER dans $50 \%$ des cas. Ces sollicitations concernaient : l'organisation du dispositif, le déroulement du DES et l'obtention de précisions quant aux relances à faire et à la nouvelle version du script à corriger. Un des responsables de CPL avait été contacté par un étudiant du fait de difficultés à joindre ou à s'entendre avec son ER.

Les difficultés exprimées par les responsables de CPL étaient les suivantes :

- temps important nécessaire au bon déroulement du dispositif ;

- nécessité d'une rigueur d'organisation et de suivi ;

- difficultés à obtenir le retour spontané des ER lors des sollicitations par mail ;

- impossibilité de vérifier la véracité des retours exprimés par les étudiants lors des cours de DES.

\section{Discussion}

Limites

Du fait de l'aspect déclaratif du recueil, nos données étaient exposées à des biais d'information, notamment celui de désirabilité sociale. Pour les ER ayant répondu par retour de courriel, leurs réponses étaient dès lors confidentielles mais non anonymes. En effet, les chercheurs avaient leurs coordonnées courriel même si le questionnaire ne faisait pas mention spécifiquement de l'identité du répondeur. Il est possible que certains aient répondu de façon à satisfaire les attentes du DMG. L'étude auprès des étudiants s'est tenue quelques mois après la fin de l'aide au premier script. Le recueil rétrospectif des données a pu générer un biais de mémorisation. Il existait également un biais de sélection, tous les étudiants et enseignants n'ayant pas participé à l'étude. Il faut, en effet, se demander si les répondeurs n'étaient pas les plus investis dans le dispositif ou au contraire, les plus revendicatifs. Il existait enfin un biais de confusion du fait que les responsables de CPL étaient également eux-mêmes des enseignants référents. Il est possible qu'en tant que responsables très impliqués, ils aient été moins objectifs pour décrire l'implication des autres ER. Compte tenu de tous ces biais, les résultats ne permettent que de générer des hypothèses. Celles-ci devraient néanmoins conduire à proposer des améliorations à l' accompagnement par les enseignants en cours ainsi qu'à d'autres travaux de recherche.

\section{Analyse}

Concernant le nombre de scripts envoyés aux ER, il existe une adéquation entre les réponses des étudiants et des enseignants. En effet, il est probable que la déclaration de sollicitation du dispositif d'aide par les étudiants soit plus importante du fait de leur meilleur taux de participation à l'étude. Quoi qu'il en soit, le niveau déclaré de sollicitation du dispositif d'aide à l'écriture du premier script était satisfaisant puisque il était supérieur à $75 \%$, chez les étudiants comme les enseignants. Par ailleurs, 71,9 \% des étudiants déclaraient y trouver un intérêt. Ces résultats confirment que la mise en place de cette aide à l'écriture du premier script a été très bien perçue. Même si elle nécessite quelques améliorations, elle n'en demeure pas moins un tutorat ponctuel à perpétuer.

\section{Difficultés rencontrées par les étudiants}

Les étudiants percevaient qu'ils étaient en difficulté au sujet de ce qui était attendu du portfolio et de l'écriture des scripts. Les ER le percevaient chez les étudiants (respectivement : 63,9 et 82,4\%). Cette 
difficulté pourrait venir du changement de paradigme pédagogique entre le deuxième et le troisième cycle des études médicales. Dorénavant le corps enseignant s'intéresse moins au savoir médical considéré comme acquis, qu'aux expériences de l'étudiant, aux difficultés qu'il a rencontrées au cours de son parcours d'apprentissage, et qui forgeront ses compétences professionnelles futures.

Une triple difficulté se présente alors aux étudiants : l'appropriation d'un nouveau mode d' apprentissage, l'intégration de la démarche réflexive comme outil d'auto-formation et l'appropriation de l'outil pédagogique qu'est le portfolio de leur DMG. Il n'est pas étonnant que la majorité d'entre eux se sentent en difficulté face à l'écriture du premier script et qu'ils soient motivés par cette initiative d'aide à son écriture.

Les étudiants avaient également émis la difficulté de trouver des situations appropriées aux soins primaires lors des stages hospitaliers. Pourtant, Snadden ${ }^{[6]}$ a mis en évidence que le portfolio peut être un bon moyen de faire le pont entre l'activité hospitalière et la pratique ambulatoire de la médecine générale. En effet, il permettrait aux étudiants de passer de l'une à l'autre en comprenant progressivement les spécificités de la médecine générale et la nécessité de compétences propres aux soins primaires. Connaissant cette difficulté, les promoteurs du dispositif bordelais ont recommandé que les scripts s'attachent à rendre compte du processus de recontextualisation aux soins primaires de la situation-problème hospitalière sélectionnée par les étudiants pour leur démarche réflexive et leurs apprentissages.

Une autre critique des étudiants était celle de thèmes imposés pour la rédaction de scripts. Driessen ${ }^{[7]}$ a mis en évidence qu'une structure flexible du portfolio était indispensable pour limiter les effets contre-productifs tels que des travaux superficiels ou falsifiés. En effet, face à des thèmes imposés, l'étudiant peut tout d'abord se sentir guidé dans son travail d'écriture mais peu à peu, s'apercevoir que les thèmes imposés ne correspondent pas à ses propres questionnements. Le risque est alors de voir apparaître des scripts rédigés uniquement dans le but de la validation et non pas dans celui d'une auto-formation de qualité.

Pour ce même auteur, les autres éléments indispensables à une mise en place du portfolio sont de communiquer clairement aux étudiants les buts du travail et les procédures de validation ${ }^{[7]}$. L'intégration du portfolio dans la validation du cursus, un temps d'écriture raisonnable et une bonne utilisation du portfolio au cours des séances d'enseignements sont nécessaires pour ne pas démotiver l'étudiant. Enfin, l'accompagnement par tutorat nécessite certes un grand investissement mais paraît crucial ${ }^{[8,9,10]}$.

Quel que soit le support utilisé et l'accompagnement mis en place, les freins à l'écriture clinique tels qu'ils sont repérés dans la littérature ${ }^{[3,11]}$ sont toujours les mêmes : le manque de temps, l'absence ou l'insuffisance pédagogique, la mauvaise qualité relationnelle avec les enseignants et le manque de clarté du contenu ou des consignes de travail.

Un étudiant évoquait également dans ses réponses ouvertes le manque d'autonomisation lié au dispositif d'aide. Une étude menée par le DMG d'Angers pointait le même problème ${ }^{[12]}$. Dans un souci de facilitation de l'appropriation par les étudiants des méthodes et des outils pédagogiques, les enseignants ne doivent pas dénaturer le paradigme dans lequel ils s'inscrivent. L'absence de cohérence entre un accompagnement trop présent ou un portfolio trop sélectif, et la visée d'une démarche de formation auto-dirigée peut dérouter les étudiants.

\section{Echanges avec les enseignants référents (ER)}

Les résultats sont discordants au sujet du temps passé avec les étudiants dans le dispositif d'aide à l'écriture du premier script. Alors que $81 \%$ des étudiants déclaraient avoir passé moins de 15 minutes avec un ER, seuls $35 \%$ des ER déclaraient ce tempslà. Quatre pour cent des étudiants déclaraient avoir passé plus d'une heure avec leur ER contre 17,9\% d'après les enseignants. Il est possible qu'il s'agisse simplement d'une appréciation différente du temps ou que les ER participants de l'étude soient ceux qui aient passé le plus de temps avec les étudiants. Enfin, nos échantillons sont numériquement faibles. Ces résultats sont donc à interpréter avec prudence mais le fait que les trois quarts des étudiants ne soient pas satisfaits du temps passé avec leur ER reflète probablement la nécessité d'une interaction plus approfondie. En effet, dans notre étude, la majorité des 
interactions ont eu lieu par mail. Cela pourrait en partie expliquer la durée inférieure à 15 minutes des échanges. Ce mode d'échange ne semble pas complètement satisfaisant. S'il peut exister, il ne doit pas être la règle, au risque d'un accompagnement de moins bonne qualité et donc de difficultés persistantes pour l'écriture des scripts suivants.

\section{Le tutorat, moyen indispensable pour une bonne implantation du porffolio}

Le rôle majeur du tutorat dans l'apprentissage par compétences est décrit dans de nombreux $\operatorname{articles}^{[8,10,13]}$. Il faciliterait la reconnaissance par l'enseignant des difficultés d'apprentissage de chaque étudiant et favoriserait l'évocation de l'aspect émotionnel au sein des scripts. Celui-ci est en effet trop souvent négligé au profit de l'aspect clinique. $\mathrm{Au}$ terme du dispositif d'aide, $81 \%$ des étudiants seraient favorables à une aide complémentaire, tout comme $88 \%$ des enseignants ayant jugé l'aide comme partiellement suffisante. Comme le suggère la littérature $^{[10,14]}$, l'accompagnement des étudiants est une condition cruciale lorsque des dispositifs pédagogiques tels que le portfolio sont implantés dans le cadre d'une approche pédagogique par compétences. Devant les difficultés du DMG à proposer un tutorat individuel tout au long du DES, cette aide individuelle précoce et ponctuelle pourrait être une solution, en complément du suivi collectif à distance. Comme le souhaitent les étudiants et les enseignants, une aide supplémentaire pourrait être envisagée. Celle-ci serait ciblée sur les internes en difficulté pour à la fois respecter l'autonomisation dans l'apprentissage de certains étudiants et répondre à des exigences d'économie en ressources humaines, en temps et en coût. Elle serait de nouveau individuelle et ponctuelle, par exemple au milieu du cursus de troisième cycle des étudiants.

Quelques études françaises ${ }^{[12,15,16]}$ ont abordé les avantages et limites du tutorat dans les DES de médecine générale. Beaucoup plébiscitent cette approche. Cependant, les effectifs d'internes s'accroissant d'année en année, le tutorat individuel deviendra de plus en plus difficile à pérenniser si les effectifs et les budgets alloués au corps enseignant n'augmentent pas proportionnellement. Certaines facultés ont introduit des tutorats collectifs et ont pu montrer que leur intérêt était croissant avec l'évolution des internes de la première à la troisième année de cursus, favorisant le sens critique et l'auto-évaluation $^{[17]}$. Le DMG de Bordeaux propose la combinaison d'une aide personnalisée en début de cursus avec des séances de tutorat collectif. Cette démarche pourrait être une solution pour d'autres DMG en France rencontrant les mêmes difficultés.

Sous réserve d'améliorer la communication entre ER et étudiant, l'initiative semblait avoir plu aux internes comme aux enseignants. Les résultats inciteraient donc à en poursuivre la mise en œuvre, avec quelques améliorations. L'aide ponctuelle à l'écriture du premier script, associée aux séances collectives, pourrait être une solution novatrice et adaptée aux besoins des étudiants, tout en tenant compte des moyens des facultés.

\section{Perspectives}

Cette étude était à la fois originale et nécessaire. En effet, même s'il s'agit d'une enquête déclarative, il était indispensable de faire ce travail exploratoire afin de valider le dispositif mis en place. Cette enquête a permis de mettre en évidence des actions efficientes mais aussi les lacunes de l'accompagnement des internes. D'autres facteurs pouvant influencer l'adhésion des internes au portfolio devraient être ultérieurement étudiés. Parmi ces facteurs, les caractéristiques des étudiants comme leur âge, leur sexe ou leur université d' origine pourraient être analysés. Du coté des enseignants, il serait pertinent d'étudier l'influence de facteurs comme : le sexe, l'âge, l'ancienneté au sein du DMG, leurs actions antérieures de chargés d'enseignement, le type d'activité libérale, le temps passé au cabinet. Ces facteurs influencent probablement la disponibilité et le temps consacré à l'aide à l'écriture du premier script. Les caractéristiques intrinsèques des enseignants devraient également être étudiées : leur propre réflexivité, leur motivation, leur sens du travail, leur identité professionnelle, leur expertise clinique et 
pédagogique $^{[18]}$. Tous ces facteurs sont des facteurs de confusion non pris en compte dans l'interprétation de nos résultats. D'autres travaux de recherche quantitatifs mais aussi qualitatifs sur les caractéristiques intrinsèques des enseignants doivent être menés, dans une logique à la fois compréhensive et évaluative de l'accompagnement des étudiants par les enseignants.

D'autres études devraient enfin évaluer l'impact de l'aide à l'écriture du premier script sur la qualité des portfolios produits en fin de DES.

Suite aux résultats de ce travail, quelques modifications ont été apportées au dispositif d'aide à l'écriture du premier script. Dès le début de leur DES, l'intérêt de la démarche réflexive, les principes de l'apprentissage par compétences et l'intérêt du portfolio sont plus explicitement exposés aux étudiants. Afin de les aider dans l'appropriation de ce nouvel outil pédagogique, le dispositif d'aide à l'écriture leur est présenté plus précocement. Pour faciliter la communication entre les enseignants et les étudiants, le DMG a mis en place l'e-portfolio, en intégrant le portfolio dans l'interface de formation disponible sur le serveur Internet de l'université. Les objectifs de l'eportfolio sont : faciliter l'interaction de l'ER et de son étudiant pour l'évaluation du premier script ; faciliter le stockage des scripts ; améliorer la visibilité de l'avancée de leur production tant pour les internes que pour les enseignants. En utilisant ce nouvel outil pédagogique, le DMG souhaite aussi améliorer la perception du portfolio par les étudiants, en rendant le processus plus ergonomique, avec un support numérique auxquels les étudiants sont habitués. Ce support numérique permet l'implémentation de liens hypertextes et d'annexes par l'étudiant, facilement accessibles pour les enseignants ${ }^{[10,14]}$. Ce nouvel outil devrait être évalué par des travaux à venir.

\section{Conclusion}

Le changement de paradigme que constitue un recentrage des dispositifs pédagogiques sur l'apprentissage des étudiants, dans une logique d'approche pédagogique par compétences nécessite que de nombreuses conditions soient réunies. Les capacités d'auto-évaluation des étudiants, même en formation spécialisée, ne doivent pas être surestimées ${ }^{[19]}$, ce qui nécessite la mise en œuvre d'une supervision et d'un accompagnement exigeants. Sans cela, la tenue d'un portfolio risque d'être très vulnérable.

L'aide individualisée à l'écriture d'un premier script, rapportée dans ce travail, été introduite dans ce sens et apporte des solutions partielles. L'adhésion au dispositif reste cependant très dépendante du lien tissé entre l'enseignant référent et l'étudiant sur le plan de l'interaction pédagogique, dans le cadre du modèle de rôle qu'elle permet de développer. La mise en place de l'e-portfolio peut sans doute, sous certaines conditions, constituer un élément facilitant, que d'autres études devront s'attacher à documenter.

\section{Contributions}

Mélanie Afonso a participé au recueil et à l'analyse des données, et à l'écriture du manuscrit. JeanLouis Demeaux a participé à l'élaboration du travail, à l'écriture et à la révision du manuscrit. Shérazade Kinouani et Bernard Gay ont participé à la relecture et à la révision du manuscrit.

\section{Déclaration d'intérêts}

Aucun auteur ne déclare de conflit d'intérêt en lien avec le contenu de cet article

\section{Approbation éthique}

\author{
Non sollicitée
}

\section{Références}

1. Jouquan J, Bail P. A quoi s'engage-t-on en basculant du paradigme d'enseignement vers le paradigme d'apprentissage ? Pédagogie Médicale 2003;4:16375. 
2. Nguyen DQ, Blais JG. Approche par objectifs ou approche par compétences? Repères conceptuels et implication pour les activités d'enseignement, d'apprentissage et d'évaluation au cours de la formation clinique. Pédagogie Médicale 2007;8:232-51.

3. Naccache N, Samson L, Jouquan J. Le portfolio en éducation des sciences de la santé un outil d'apprentissage, de développement professionnel et d'évaluation. Pédagogie Médicale 2006;7:110-27.

4. Kinouani S. Analyse du portfolio comme outil de formation et d'évaluation des étudiants en médecine générale de 2005 à 2012 à l'Université de Bordeaux Mémoire Diplôme Universitaire de Pédagogie. Université de Bordeaux, 2012.

5. Afonso M. Evaluation du dispositif d'aide à l'écriture du premier script du portfolio en troisième cycle de Médecine Générale. Etude observationnelle réalisée au cours de l'année universitaire 2011-2012 auprès des étudiants de TCEM1 et des enseignants de médecine générale de l'Université de Bordeaux. Thèse pour le Diplôme d'état de doctorat en médecine, Université de Bordeaux, 2013.

6. Snadden $\mathrm{D}$, Thomas ML. Portfolio learning in general practice vocational training- Does it work? Med Educ 1998;32:401-6.

7. Driessen EW, Overeem K, Van Tartwijk J,Van der Leuten PM, Muijtjens AMM. Validity of portfolio assessment: which qualities determine ratings? Med Educ 2006;40:862-6.

8. Driessen EW,Van Tartwijk J,Van der Leuten PM, Wass Val J. Portfolio in medical education: why do they meet with mixed success? A systematic review. Med Educ 2007;41:1224-33.

9. Van Tartwijk J, Driessen E, Stokking K, Van der Vleuten C. Factors influencing the successful introduction of portfolios. Qual Higher Educ 2007;13:69-79.

10. Van Tartwijk J, Driessen EW. Portfolios for assessment and learning: AMEE Guide no. 45 Med Teach 2009;31:790-801.

11. Mathers NJ, Challis MC, Howe AC, Field NJ. Portfolios in continuing medical education - effective and efficient? Med Educ 1999;33:521-530.
12. Acker I, Foucat J, Cailliez E, Huez JF, Joly-Guillou ML, Bouzille G. Evaluation de l'utilisation du Portfolio par les internes en médecine générale d'Angers. Exercer 2012;102:121-6.

13. Driessen EW, Tartwijck J, Dornan T. The self-critical doctor: helping students become more reflective. BMJ 2008;338:827-30.

14. Tochel C, Haig A, Hesketh A, Cadzow A, Beggs K, Colthart I, Peacock H. The effectiveness of portfolios for post-graduate assessment and education: BEME Guide $\mathrm{n}^{\circ} 12$ Med Teach 2009;31:299-318.

15. Le Mauff P, Pottier P, Goronflot L, Barrier J. Evaluation d'un dispositif expérimental d'évaluation certificative des étudiants en fin de 3ème cycle de Médecine Générale. Pédagogie Médicale 2006;7: 142-54.

16. Chalons B. Quelles sont les difficultés et les bénéfices du RSCA perçus par les internes inscrits en DES de Médecine Générale à la faculté de Tours au cours du second semestre de l'année universitaire 2008-2009 ? Thèse pour le Diplôme d'état de doctorat en médecine, Université de Tours, 2011.

17. Jami A, Saint Lary O, Bernard E, Saunier de Almeida A, Lefranc-Morin C, Ouzana A. Perceptions et représentations des internes de médecine générale à l'égard d'un outil pédagogique issu du paradigme d'apprentissage. Pédagogie Médicale 2012;13:2737.

18. Chamberland M, Hivon R. Les compétences de l'enseignant clinicien et le modèle de rôle. Pédagogie Médicale 2005;6:98-111

19. Dory V, de Foy T, Degryse J. L'auto-évaluation : postulat préalable, finalité de la mission éducative ou utopie pédagogique ? Clarifications conceptuelles et pistes pour une application en éducation médicale. Pédagogie Médicale 2009;10:41-53.

Correspondance et offprints : Mélanie Afonso. Université de Bordeaux, Département de Médecine Générale, 146 rue Léo Saignat Case 148, 33076 Bordeaux Cedex, France. Mailto : melanie.afonso@u-bordeaux2.fr 